\title{
Multiple positive solutions of a semipositone singular boundary value problem on time scales
}

\section{Rajendra Dahal ${ }^{*}$}

\section{"Correspondence:}

rdahal@coastal.edu

Department of Mathematics and

Statistics, Coastal Carolina

University, Conway, SC 29526, USA

\begin{abstract}
In this paper, we study the following singular semipositone boundary value problem on time scales:

$$
\left\{\begin{array}{l}
-x^{\Delta \nabla}=p(t) f(t, x)+q(t), \quad t \in(\rho(a), \sigma(b))_{\mathbb{T}}, \\
x(\rho(a))=0, \quad x(\sigma(b))=0,
\end{array}\right.
$$

where $p:(\rho(a), \sigma(b))_{\mathbb{T}} \rightarrow[0, \infty)$ and $f:[\rho(a), \sigma(b)]_{\mathbb{T}} \rightarrow[0, \infty)$ are continuous; and $q:(\rho(a), \sigma(b))_{\mathbb{T}} \rightarrow(-\infty, \infty)$ is Lebesgue $\nabla$-integrable. By constructing a special cone and using a fixed point theorem, we establish some sufficient conditions for the existence of multiple positive solutions. Two examples are given at the end of the paper to demonstrate our result.
\end{abstract}

MSC: 39A10; 39A13; 34B16; 34B18; 34N05

Keywords: time scales; positive solutions; semipositone; cone; fixed point theorem; Green's function

\section{Introduction}

The theory of dynamic equations on time scales has been studied by many mathematicians since it not only provides a unifying structure of differential and difference equations, but also it has led to many physical, chemical and biological applications such as insect population models, neural networks, heat transfer, epidemic models. In this paper we study the existence of positive solutions of the following singular semipositone boundary value problem (BVP):

$$
\left\{\begin{array}{l}
-x^{\Delta \nabla}=p(t) f(t, x)+q(t), \quad t \in(\rho(a), \sigma(b))_{\mathbb{T}} \\
x(\rho(a))=0, \quad x(\sigma(b))=0
\end{array}\right.
$$

where $\mathbb{T}$ is a time scale; $p:(\rho(a), \sigma(b))_{\mathbb{T}} \rightarrow[0, \infty)$ and $f:[\rho(a), \sigma(b)]_{\mathbb{T}} \times[0, \infty) \rightarrow[0, \infty)$ are continuous; and $q:(\rho(a), \sigma(b))_{\mathbb{T}} \rightarrow(-\infty, \infty)$ is Lebesgue $\nabla$-integrable. Note that $(a, b)_{\mathbb{T}}$ denotes the time scale interval, $(a, b) \cap \mathbb{T}$. The study of analysis on time scales was initiated by Stefan Hilger in 1988, and the first paper appearing in this field by Hilger was [1]. An excellent introduction to time scales calculus can be found in Chapter 1 of [2]

\section{空 Springer}

○2013 Dahal; licensee Springer. This is an Open Access article distributed under the terms of the Creative Commons Attribution License (http://creativecommons.org/licenses/by/2.0), which permits unrestricted use, distribution, and reproduction in any medium, provided the original work is properly cited. 
and in [3]. Chapters 7 and 9 of the text [3] deal with finding positive solutions of several boundary value problems on time scales by various contemporary authors. We also want to guide the readers to take a look at early research papers on time scales [4-6]. Note that in our problem, $q$ may change its sign, so we call this type of problem semipositone. The study of nonlinear, singular boundary value problems is not new but the consideration of a semipositone case is relatively new even in differential equations. Semipositone problems arise in many physical and chemical processes such as in chemical reactor theory [7]. In applications one is interested in finding positive solutions. In recent years, several authors studied semipositone BVPs on time scales, and we want to mention some papers in literature [8-14], and the references therein. Among several other papers in difference equations, Bai and Xu [15] recently studied semipositone problems in difference equations. All the above mentioned papers are concerned with the existence of only one positive solution. Anderson and Wong in [8] and Bai and Xu in [15] also require that the nonlinear term of the equation has a finite lower bound. But we do not require that in this paper and, in fact, the nonlinear term is allowed to decrease without bound. Thus this paper fills the gap in literature on time scales calculus providing the existence of multiple positive solutions and allowing the nonlinear term decrease without bound at the same time. To our best knowledge, this result is new in the time scales setting and it covers the results not only for ordinary differential equations but also difference equations, $q$-difference equations, and other exotic time scales. The nabla derivative was introduced in [16]. As a special case when $\mathbb{T}=\mathbb{R}$, this result includes those of [17]. Let $a$ and $b$ be such that $0 \leq \rho(a) \leq a<b \leq \sigma(b)<\infty$, and $(\rho(a), \sigma(b))_{\mathbb{T}}$ has at least two points.

\section{Preliminaries and lemmas}

We first find the related Green's function of our BVP. The Green's function for the BVP

$$
-x^{\Delta \nabla}=0, \quad x(\rho(a))=0=x(\sigma(b))
$$

is given by [16]

$$
G(t, s)=\frac{1}{\sigma(b)-\rho(a)} \begin{cases}(s-\rho(a))(\sigma(b)-t), & \text { for } s \leq t \\ (t-\rho(a))(\sigma(b)-s), & \text { for } t \leq s .\end{cases}
$$

For convenience, we let

$$
e(t):=\frac{(t-\rho(a))(\sigma(b)-t)}{(\sigma(b)-\rho(a))^{2}}=\frac{G(t, t)}{\sigma(b)-\rho(a)},
$$

then it is easy to see

$$
e(t) G(s, s) \leq G(t, s) \leq G(s, s) \quad \text { or } \quad G(t, t) .
$$

Define

$$
c:=\inf \left\{t \in \mathbb{T}: t \geq \frac{(\sigma(b)-\rho(a))}{4}\right\}, \quad d:=\sup \left\{t \in \mathbb{T}: t \leq \frac{3(\sigma(b)-\rho(a))}{4}\right\} .
$$


Next we set

$$
\alpha:=\min _{t \in[c, d]_{\mathbb{T}}} e(t)
$$

Then we have

$$
G(t, s) \geq \alpha G(s, s)>0, \quad t \in[c, d]_{\mathbb{T}} .
$$

Next we want to construct a cone in which we will look for positive solutions.

Let $X:=\left\{x \in C\left([\rho(a), \sigma(b)]_{\mathbb{T}}, \mathbb{R}\right)\right\}$, with $\|x\|=\sup _{t \in[\rho(a), \sigma(b)]_{\mathbb{T}}}|x(t)|$, and define $P:=\{x \in P$ : $\left.x(t) \geq\|x\| e(t), t \in[\rho(a), \sigma(b)]_{\mathbb{T}}\right\}$.

Then one can easily verify that $X$ is a real Banach space, and $P$ is a cone in $X$.

Now we state the well-known fixed point theorem that we will use later in this paper.

Theorem 1 [18] Let $X$ be a real Banach space, and let $P \subset X$ be a cone. Let $\Omega_{1}, \Omega_{2}$ be two bounded open subsets of $X$ with $0 \subset \Omega_{1}, \bar{\Omega}_{1} \subset \Omega_{2}$. Assume that $A: P \cap\left(\bar{\Omega}_{2} \backslash \Omega_{1}\right) \rightarrow P$ is a completely continuous operator such that either

(i) $\|A u\| \leq\|u\|$ for all $u \in P \cap \partial \Omega_{1}$ and $\|A u\| \geq\|u\|$ for all $u \in P \cap \partial \Omega_{2}$, or

(ii) $\|A u\| \geq\|u\|$ for all $u \in P \cap \partial \Omega_{1}$ and $\|A u\| \leq\|u\|$ for all $u \in P \cap \partial \Omega_{2}$.

Then $A$ has a fixed point in $P \cap\left(\bar{\Omega}_{2} \backslash \Omega_{1}\right)$.

As usual with a semipositone problem, we want to consider the following singular auxiliary problem:

$$
-w^{\Delta \nabla}=q^{-}(t), \quad w(\rho(a))=0, \quad w(\sigma(b))=0,
$$

where $q^{ \pm}(t):=\max \{ \pm q(t), 0\}$. Its unique solution is given by (see [12] for proof)

$$
w(t)=\int_{\rho(a)}^{b} G(t, s) q^{-}(s) \nabla s
$$

Consider the following singular positone BVP:

$$
\left\{\begin{array}{l}
z^{\Delta \nabla}(t)+p(t) f\left(t,[z-w]^{+}(t)\right)+q^{+}(t)=0, \quad t \in(\rho(a), \sigma(b))_{\mathbb{T}}, \\
z(\rho(a))=0, \quad z(\sigma(b))=0 .
\end{array}\right.
$$

Next define an integral operator $T: P \rightarrow X$ by

$$
(T z)(t)=\int_{\rho(a)}^{b} G(t, s)\left[p(s) f\left(s,[z-w]^{+}(s)\right)+q^{+}(s)\right] \nabla s .
$$

It is easy to see that finding solutions of BVP (9) is equivalent to finding fixed points of the operator $T$ on $P$.

Now we state and prove a lemma that connects singular positone BVP (9) to main BVP (1).

Lemma 2 If $z(t)$ is the unique positive solution of singular positone BVP (9) such that $z(t) \geq$ $w(t)$ then $B V P(1)$ has a positive solution $x(t):=z(t)-w(t)$. 
Proof Let $z(t)=x(t)+w(t), t \in[\rho(a), \sigma(b)]_{\mathbb{T}}$. Then, by the first equation of (9), it follows that

$$
\begin{gathered}
x^{\Delta \nabla}(t)+w^{\Delta \nabla}(t)+p(t) f(t,[z-w](t))+q^{+}(t)=0 \\
\Longrightarrow \quad x^{\Delta \nabla}(t)-q^{-}(t)+p(t) f(t, x(t))+q^{+}(t)=0 \\
\Longrightarrow \quad x^{\Delta \nabla}(t)+p(t) f(t, x(t))+q(t)=0 .
\end{gathered}
$$

Also,

$$
\begin{aligned}
& x(\rho(a))=z(\rho(a))-w(\rho(a))=0, \\
& x(\sigma(b))=z(\sigma(b))-w(\sigma(b))=0 .
\end{aligned}
$$

Now we impose the following conditions for the rest of the paper:

(H1) $p:(\rho(a), \sigma(b))_{\mathbb{T}} \times[0, \infty) \rightarrow[0, \infty)$ and $f:[\rho(a), \sigma(b)]_{\mathbb{T}} \times[0, \infty) \rightarrow[0, \infty)$ are continuous.

(H2) $q:(\rho(a), \sigma(b))_{\mathbb{T}} \rightarrow(-\infty, \infty)$ is Lebesgue $\nabla$-integrable such that

$$
0<\int_{\rho(a)}^{b} G(s, s)\left[p(s)+q^{+}(s)\right] \nabla s=: L<2 r
$$

where $\int_{\rho(a)}^{b}(\sigma(b)-\rho(a)) q^{-}(s) \nabla s=: r>0$.

(H3) There exists $R>2 r$ such that for $(t, x) \in[\rho(a), \sigma(b)]_{\mathbb{T}} \times\left[\frac{\alpha}{2} R, R\right]$,

$$
f(t, x) \geq \frac{R}{\alpha l}, \quad \text { where } l:=\int_{c}^{d} G(s, s) p(s) \nabla s \text {, and } \alpha \text { is as defined in (5). }
$$

(H4) There exists $R_{1}>\max \left\{R,\left(\frac{R}{\alpha l}+1\right) L\right\}$ such that for $(t, x) \in[\rho(a), \sigma(b)]_{\mathbb{T}} \times\left[0, R_{1}\right]$,

$$
f(t, x) \leq \frac{R_{1}}{L}
$$

(H5) $\lim _{x \rightarrow \infty} \frac{f(t, x)}{x}=+\infty$ uniformly for $t$ in any closed subinterval of $(\rho(a), \sigma(b))_{\mathbb{T}}$. To apply Theorem 1, we first prove the following lemmas.

Lemma 3 Assume that $(\mathrm{H} 1)$ and $(\mathrm{H} 2)$ hold. Then $T: P \rightarrow P$ is completely continuous.

Proof First we show $T: P \rightarrow P$. Let $z \in P$ and $t \in[\rho(a), \sigma(b)]_{\mathbb{T}}$. Then

$$
\begin{aligned}
&(T z)(t)=\int_{\rho(a)}^{b} G(t, s)\left[p(s) f\left(s,[z-w]^{+}(s)\right)+q^{+}(s)\right] \nabla s, \\
&(T z)(t) \leq \int_{\rho(a)}^{b} G(s, s)\left[p(s) f\left(s,[z-w]^{+}(s)\right)+q^{+}(s)\right] \nabla s \\
& \Longrightarrow \quad \int_{\rho(a)}^{b} G(s, s)\left[p(s) f\left(s,[z-w]^{+}(s)\right)+q^{+}(s)\right] \nabla \geq\|T z\| .
\end{aligned}
$$


Then, using (4), we get

$$
\begin{aligned}
&(T z)(t)=\int_{\rho(a)}^{b} G(t, s)\left[p(s) f\left(s,[z-w]^{+}(s)\right)+q^{+}(s)\right] \nabla s, \\
&(T z)(t) \geq e(t) \int_{\rho(a)}^{b} G(s, s)\left[p(s) f\left(s,[z-w]^{+}(s)\right)+q^{+}(s)\right] \nabla s \\
& \Longrightarrow \quad(T z)(t) \geq e(t)\|T z\| .
\end{aligned}
$$

Next let $B \subset P$ be any bounded set, then for any $z \in B$, there exists a constant $M>0$ such that $\|z\| \leq M$. Now, for any $z \in B$ and $s \in[\rho(a), \sigma(b)]_{\mathbb{T}}$, we have

$$
[z-w]^{+}(s) \leq z(s) \leq\|z\| \leq M
$$

and

$$
\begin{aligned}
\|(T z)(t)\| & =\max _{t \in[\rho(a), \sigma(b)]_{\mathbb{T}}} \int_{\rho(a)}^{b} G(t, s)\left[p(s) f\left(s,[z-w]^{+}(s)\right)+q^{+}(s)\right] \nabla s \\
& \leq \int_{\rho(a)}^{b} G(s, s)\left(M_{1}+1\right)\left[p(s)+q^{+}(s)\right] \nabla s \\
& \leq\left(M_{1}+1\right) \int_{\rho(a)}^{b} G(s, s)\left[p(s)+q^{+}(s)\right] \nabla s<\infty, \quad \text { by }(\mathrm{H} 2),
\end{aligned}
$$

where

$$
M_{1}:=\max _{[\rho(a), \sigma(b)]_{\mathbb{T}} \times[0, M]} f(t, x) .
$$

Therefore $T(B)$ is uniformly bounded.

By standard arguments (see [12]) using the Arzela-Ascoli theorem and the Lebesgue dominated convergence theorem, we can easily see that $T$ is a completely continuous operator.

Lemma 4 Assume that (H1)-(H3) hold, and set $\Omega_{R}=\{z \in P:\|z\|<R\}$ and $\partial \Omega_{R}=\{z \in P$ : $\|z\|=R\}$. Then $\|T z\| \geq\|z\|$ for all $z \in P \cap \partial \Omega_{R}$, where $R>2 r$ is as given in $(\mathrm{H} 3)$.

Proof For any $z \in \partial \Omega_{R}, t \in[c, d]_{\mathbb{T}}$, we have

$$
\begin{aligned}
z(t)-w(t) & =z(t)-\int_{\rho(a)}^{b} G(t, s) q^{-}(s) \nabla s \\
& \geq z(t)-\frac{G(t, t)}{(\sigma(b)-\rho(a))} \int_{\rho(a)}^{b}(\sigma(b)-\rho(a)) q^{-}(s) \nabla s \\
& =z(t)-e(t) r \geq z(t)-\frac{z(t)}{\|z\|} r=z(t)-\frac{r}{R} z(t) \geq \frac{1}{2} z(t) \\
& \geq \frac{1}{2} e(t)\|z\| \geq \frac{1}{2} \min _{t \in[c, d]_{\mathbb{T}}} e(t) R=\frac{\alpha}{2} R>0 .
\end{aligned}
$$


So, for any $z \in \partial \Omega_{R}$ and $t \in[c, d]_{\mathbb{T}}$, we have

$$
\frac{\alpha}{2} R \leq z(t)-w(t) \leq R
$$

Then, using (H3), we get

$$
\begin{aligned}
\|T z\| & \geq(T z)(t)=\int_{\rho(a)}^{b} G(t, s)\left[p(s) f\left(s,[z-w]^{+}(s)\right)+q^{+}(s)\right] \nabla s \\
& \geq \int_{c}^{d} G(t, s) p(s) f(s, z(s)-w(s)) \nabla s \\
& \geq \frac{R}{\alpha l} \int_{c}^{d} G(t, s) p(s) \nabla s \\
& \geq \frac{R}{\alpha l} \int_{c}^{d} \alpha G(s, s) p(s) \nabla s=\frac{R}{l} l=\|z\| .
\end{aligned}
$$

Thus we have $\|T z\| \geq\|z\|$ for all $z \in P \cap \partial \Omega_{R}$.

Lemma 5 Assume that (H1)-(H5) hold, and set $\Omega_{R_{1}}=\left\{z \in P:\|z\|<R_{1}\right\}$. Then $\|T z\| \leq\|z\|$ for all $z \in P \cap \partial \Omega_{R_{1}}$, where $R_{1}$ is as given in $(\mathrm{H} 4)$.

Proof From (H4) we have that $R_{1}>R>2 r>0$. Note that for any $z \in \partial \Omega_{R_{1}}$ and $s \in$ $[\rho(a), \sigma(b)]_{\mathbb{T}}$,

$$
0 \leq[z-w]^{+}(s) \leq z(s) \leq\|z\|=R_{1} .
$$

Now, for any $z \in \partial \Omega_{R_{1}}, t[\rho(a), \sigma(b)]_{\mathbb{T}}$,

$$
\begin{aligned}
\|T z\| & =\max _{t \in[\rho(a), \sigma(b)]_{\mathbb{T}}} \int_{\rho(a)}^{b} G(t, s)\left[p(s) f\left(s,[z-w]^{+}(s)\right)+q^{+}(s)\right] \nabla s \\
& \leq \int_{\rho(a)}^{b} G(s, s)\left[p(s) f(s, z(s)-w(s))+q^{+}(s)\right] \nabla s \\
& \leq \frac{R_{1}}{L} \int_{\rho(a)}^{b} G(s, s)\left[p(s)+q^{+}(s)\right] \nabla s \\
& =R_{1}=\|z\| .
\end{aligned}
$$

Thus we have $\|T z\| \leq\|z\|, z \in P \cap \partial \Omega_{R_{1}}$.

Now choose constants $\delta, \beta$ and a real number $K>0$ such that

$$
[\delta, \beta]_{\mathbb{T}} \in(\rho(a), \sigma(b))_{\mathbb{T}}
$$

and

$$
1 \leq \frac{1}{2} \frac{(\delta-\rho(a))^{2}(\sigma(b)-\beta)^{2}}{(\sigma(b)-\rho(a))^{4}} K \int_{\alpha}^{\beta} G(s, s) p(s) \nabla s .
$$


Note that using (H5), we find $\widetilde{R}>R_{1}$ such that for any $t \in[\delta, \beta]_{\mathbb{T}}$ and $x \geq \widetilde{R}$,

$$
f(t, x) \geq K x
$$

Take $R_{2}=\max \left\{\frac{2(\sigma(b)-\rho(a))^{2} \widetilde{R}}{(\delta-\rho(a))(\sigma(b)-\beta)}, \widetilde{R}\right\}$. Then we have $R_{2}>\widetilde{R}>R_{1}>R>2 r$.

Lemma 6 Assume that (H1)-(H5) hold, and set $\Omega_{R_{2}}=\left\{z \in P:\|z\|<R_{2}\right\}$. Then $\|T z\| \geq\|z\|$, $z \in P \cap \partial \Omega_{R_{2}}$.

Proof For any $z \in \partial \Omega_{R_{2}}, t \in[\delta, \beta]_{\mathbb{T}}$, we have

$$
\begin{aligned}
z(t)-w(t) & \geq z(t)-\int_{\rho(a)}^{b} G(t, t) q^{-}(s) \nabla s \\
& =z(t)-\frac{G(t, t)}{(\sigma(b)-\rho(a))} \int_{\rho(a)}^{b}(\sigma(b)-\rho(a)) q^{-}(s) \nabla s \\
& =z(t)-e(t) r \geq z(t)-\frac{z(t)}{\|z\|} r=z(t)-\frac{r}{R_{2}} z(t) \geq \frac{1}{2} z(t) \\
& \geq \frac{1}{2} e(t)\|z\| \geq \frac{1}{2} \frac{(\delta-\rho(a))(\sigma(b)-\beta)}{(\sigma(b)-\rho(a))^{2}} R_{2} \geq \widetilde{R}>0 .
\end{aligned}
$$

Then, for any $z \in P \cap \partial \Omega_{R_{2}}$ and $t \in[\delta, \beta]_{\mathbb{T}}$, we have

$$
\begin{aligned}
(T z)(t) & =\int_{\rho(a)}^{b} G(t, s)\left[p(s) f\left(s,[z-w]^{+}(s)\right)+q^{+}(s)\right] \nabla s \\
& \geq \int_{\rho(a)}^{b} G(t, s) p(s) f\left(s,[z-w]^{+}(s)\right) \nabla s \\
& \geq \int_{\delta}^{\beta} G(t, s) p(s) f(s, z(s)-w(s)) \nabla s \\
& \geq \int_{\delta}^{\beta} G(t, s) p(s) K[z(s)-w(s)] \nabla s, \quad \text { using }(12), \\
& \geq \frac{1}{2} \frac{(\delta-\rho(a))(\sigma(b)-\beta)}{(\sigma(b)-\rho(a))^{2}} K R_{2} \int_{\delta}^{\beta} G(t, s) p(s) \nabla s \\
& \geq \frac{1}{2} \frac{(\delta-\rho(a))(\sigma(b)-\beta)}{(\sigma(b)-\rho(a))^{2}} K R_{2} e(t) \int_{\delta}^{\beta} G(s, s) p(s) \nabla s \\
& \geq \frac{1}{2} \frac{(\delta-\rho(a))^{2}(\sigma(b)-\beta)^{2}}{(\sigma(b)-\rho(a))^{4}} K R_{2} \int_{\delta}^{\beta} G(t, s) p(s) \nabla s \geq R_{2}, \quad \text { using }(11) .
\end{aligned}
$$

Thus $\|T z\| \geq\|z\|$ for all $z \in P \cap \partial \Omega_{R_{2}}$.

\section{Main result}

Theorem 7 Suppose that (H1)-(H5) hold. Then $T$ has two fixed points $z_{1}(t) \in P \cap\left(\bar{\Omega}_{R_{1}} \backslash\right.$ $\left.\Omega_{R}\right)$ and $z_{2}(t) \in P \cap\left(\bar{\Omega}_{R_{2}} \backslash \Omega_{R_{1}}\right)$ such that $R \leq\left\|z_{1}\right\| \leq R_{1} \leq\left\|z_{2}\right\| \leq R_{2}$, where $R>2 r$, from (H3), and $r=\int_{\rho(a)}^{b}(\sigma(b)-\rho(a)) q^{-}(s) \nabla s$, as defined in $(\mathrm{H} 2)$. 
Proof By the previous three lemmas (Lemmas 4, 5, 6) and the cone compression/expansion theorem (Theorem 1), we get that

$$
2 r<R_{1} \leq\left\|z_{1}\right\| \leq R_{1} \leq\left\|z_{2}\right\| \leq R_{2} .
$$

Now we have

$$
\begin{aligned}
z_{1}(t)-w(t) & \geq e(t)\left\|z_{1}\right\|-\int_{\rho(a)}^{b} G(t, s) q^{-}(s) \nabla s \\
& \geq e(t)\left\|z_{1}\right\|-\int_{\rho(a)}^{b} G(t, t) q^{-}(s) \nabla s \\
& =e(t)\left\|z_{1}\right\|-e(t) r=e(t)\left(\left\|z_{1}\right\|-r \|\right)>0,
\end{aligned}
$$

and

$$
\begin{aligned}
z_{2}(t)-w(t) & \geq z_{2}-\int_{\rho(a)}^{b} G(t, s) q^{-}(s) \nabla s \\
& \geq z_{2}-\int_{\rho(a)}^{b} G(t, t) q^{-}(s) \nabla s \\
& =z_{2}-e(t) r \geq z_{2}-\frac{z_{2}}{\left\|z_{2}\right\|} r \geq z_{2}-\frac{r}{R_{1}} z_{2} \geq \frac{1}{2} z_{2}(t) \\
& \geq \frac{1}{2} e(t)\left\|z_{2}\right\| \geq \frac{1}{2} e(t) R_{1}>0 .
\end{aligned}
$$

Let $x_{1}(t)=z_{1}(t)-w(t), x_{2}(t)=z_{2}(t)-w(t)$, then by Lemma 2 , we have that

$$
x_{1}(t)=z_{1}(t)-w(t)>0, \quad x_{2}(t)=z_{2}(t)-w(t)>0, \quad t \in(\rho(a), \sigma(b))_{\mathbb{T}}
$$

are two positive solutions of BVP (1).

\section{Examples}

In this section we give two examples as applications of Theorem 7.

Example 1 Let $\mathbb{T}=$ the Cantor set. (See pages 18-19 of [2] for more information regarding this time scale.) Consider the following BVP:

$$
\left\{\begin{array}{l}
-x^{\Delta \nabla}=\frac{f(x)}{3(1-t)}-\frac{1}{\sqrt{t}+\sqrt{\rho(t)}}, \quad t \in(0,1)_{\mathbb{T}}, \\
x(0)=0, \quad x(1)=0
\end{array}\right.
$$

where $f(x)$ is defined as

$$
f(x)= \begin{cases}x^{2}, & 0 \leq x \leq 1 \\ 901 x-900, & 1 \leq x \leq 3, \\ 1,803, & 3 \leq x \leq 32 \\ \frac{11}{160} x+1,800.8, & 32 \leq x \leq 352, \\ x^{2}-122,079, & x \geq 352 .\end{cases}
$$


This BVP has at least two positive solutions as it satisfies all the hypotheses of this paper. Note that we have

$$
p(t)=\frac{1}{3(1-t)}, \quad q^{+}(t) \equiv 0, \quad q^{-}(t)=\frac{1}{\sqrt{t}+\sqrt{\rho(t)}} .
$$

Now

$$
\begin{aligned}
r & =\int_{\rho(a)}^{b}(\sigma(b)-\rho(a)) q^{-}(s) \nabla s \\
& =\int_{0}^{1} \frac{1}{\sqrt{s}+\sqrt{\rho(s)}} \nabla s=\int_{0}^{1}(\sqrt{s})^{\nabla} \nabla s=1 .
\end{aligned}
$$

In [19] the authors show that $\int_{0}^{1} \Delta t=\frac{3}{7}$, where $t \in \mathbb{T}$, the Cantor set. Using similar arguments, we get that $\int_{0}^{1} \nabla t=\frac{4}{7}$, which we use below. We have

$$
\begin{aligned}
L & =\int_{0}^{1} s(1-s)\left[\frac{1}{3(1-s)}+0\right] \nabla s \\
& =\frac{1}{3} \int_{0}^{1} s \nabla s=\frac{1}{3} \cdot \frac{4}{7}=\frac{4}{21}<2 r=2 .
\end{aligned}
$$

Similarly,

$$
l=\int_{1 / 4}^{3 / 4} s(1-s) \frac{1}{3(1-s)} \nabla s=\frac{2}{21} .
$$

Note that $\alpha=\min _{t \in[1 / 4,3 / 4]_{\mathbb{T}}} e(t)=\frac{3}{16}$. Let $R=32>2 r=2$, then for any $x \in[3,32]$, we have

$$
f(t, x)=1,803 \geq \frac{32}{3 / 16 \cdot 2 / 21}=1,792 .
$$

On the other hand, let

$$
R_{1}:=352>\left(\frac{32}{3 / 16 \cdot 2 / 21}+1\right) \cdot \frac{4}{21}=\frac{7,172}{21},
$$

then for any $x \in[0,352]$, we have $f(t, x) \leq 1,825 \leq \frac{R_{1}}{L}=1,848$.

It is clear that $\lim _{x \rightarrow \infty} \frac{f(t, x)}{x}=\infty$.

Example 2 Let $\mathbb{T}=\left\{\frac{1}{2^{n}}\right\}_{n=0}^{\infty} \cup\{0\} \cup\left\{\frac{3}{4}\right\}$. Consider the following BVP:

$$
\left\{\begin{array}{l}
-x^{\Delta \nabla}=\frac{f(x)}{1-t}-\frac{1}{\sqrt{t}}, \quad t \in(0,1)_{\mathbb{T}}, \\
x(0)=0, \quad x(1)=0,
\end{array}\right.
$$

where $f(x)$ is defined as

$$
f(x)= \begin{cases}183 x, & 0 \leq x \leq 3 \\ 549, & 3 \leq x \leq 32 \\ \frac{1}{2} x+533, & 32 \leq x \leq 232 \\ x^{2}-53,175, & x \geq 232\end{cases}
$$


This BVP has at least two positive solutions as it satisfies all the hypotheses of this paper. Note that we have

$$
p(t)=\frac{1}{1-t}, \quad q^{+}(t) \equiv 0, \quad q^{-}(t)=\frac{1}{\sqrt{t}} .
$$

Now

$$
\begin{aligned}
r & =\int_{\rho(a)}^{b}(\sigma(b)-\rho(a)) q^{-}(s) \nabla s=\int_{0}^{3 / 4} \frac{1}{\sqrt{s}} \nabla s \\
& =\int_{0}^{1 / 2} \frac{1}{\sqrt{s}} \nabla s+\int_{1 / 2}^{3 / 4} \frac{1}{\sqrt{s}} \nabla s \\
& =\left(\sqrt{2} \cdot \frac{1}{4}+2 \cdot \frac{1}{8}+2 \sqrt{2} \cdot \frac{1}{16}+\cdots\right)+\frac{2}{\sqrt{3}} \cdot \frac{1}{4} \\
& =\frac{\sqrt{2} / 4}{1-(1 / \sqrt{2})}+\frac{1}{2 \sqrt{3}} \approx 1.4,
\end{aligned}
$$

and we have

$$
\begin{aligned}
L & =\int_{0}^{3 / 4} s(1-s)\left[\frac{1}{1-s}+0\right] \nabla s=\int_{0}^{3 / 4} s \nabla s \\
& =\frac{3}{4} \cdot \frac{1}{4}+\left(\frac{1}{2} \cdot \frac{1}{4}+\frac{1}{4} \cdot \frac{1}{8}+\cdots\right)=\frac{17}{48}<2 r=2.8
\end{aligned}
$$

similarly,

$$
l=\int_{1 / 4}^{3 / 4} s(1-s) \frac{1}{(1-s)} \nabla s=\int_{1 / 4}^{3 / 4} s \nabla s=\frac{5}{16} .
$$

Note that $\alpha=\min _{t \in[1 / 4,3 / 4]_{\mathbb{T}}} e(t)=\frac{3}{16}$. Let $R=32>2 r=2.8$, then for any $x \in[3,32]$, we have

$$
f(t, x)=549 \geq \frac{32}{3 / 16 \cdot 5 / 16} \approx 546.13
$$

On the other hand, let

$$
R_{1}:=232>\left(\frac{32}{3 / 16 \cdot 5 / 16}+1\right) \cdot \frac{17}{48} \approx 193.7
$$

then for any $x \in[0,232]$, we have $f(t, x) \leq 649 \leq \frac{R_{1}}{L} \approx 655.1$.

Note it is clear that $\lim _{x \rightarrow \infty} \frac{f(t, x)}{x}=\infty$.

The author declares that he has no competing interests.

\section{Author's contributions}

The sole author has made all contributions.

\section{Acknowledgements}

The author would like to thank the anonymous referee for very helpful comments and suggestions. 


\section{References}

1. Hilger, S: Analysis on measure chains - a unified approach to continuous and discrete calculus. Results Math. 18 18-56 (1990)

2. Bohner, M, Peterson, A: Dynamic Equations on Time Scales: An Introduction with Applications. Birkhäuser, Boston (2001)

3. Bohner, M, Peterson, A: Advances in Dynamic Equations on Time Scales. Birkhäuser, Boston (2003)

4. Agarwal, RP, O'Regan, D: Triple solutions to boundary value problems on time scales. Appl. Math. Lett. 13(4), 7-11 (2000)

5. Akin, E: Boundary value problems for a differential equation on a measure chain. Panam. Math. J. 10(3), 17-30 (2000)

6. Stehlik, P: Periodic boundary value problems on time scales. Adv. Differ. Equ. 2005(1), 81-92 (2005)

7. Aris, R: Introduction to the Analysis of Chemical Reactors. Prentice Hall, Englewood Cliffs (1965)

8. Anderson, DR, Wong, PJY: Positive solutions for second-order semipositone problems on time scales. Comput. Math. Appl. 58(2), 281-291 (2009)

9. Anderson, DR, Zhai, C: Positive solutions to semi-positone second-order three-point problems on time scales. Appl. Math. Comput. 215, 3713-3720 (2010)

10. Anuradha, A, Hai, DD, Shivaji, R: Existence results for superlinear semipositone BVPs. Proc. Am. Math. Soc. 124(3), 757-763 (1996)

11. Goodrich, C: Existence of a positive solution to a nonlocal semipositone boundary value problem on a time scale. Comment. Math. Univ. Carol. 54(4), 509-525 (2013)

12. Dahal, R: Positive solutions of semipositone singular Dirichlet dynamic boundary value problems. Nonlinear Dyn Syst. Theory 9(4), 361-374 (2009)

13. Dahal, R: Positive solutions for a second-order, singular semipositone dynamic boundary value problem. Int. J. Dyn. Syst. Differ. Equ. 3(1-2), 178-188 (2011)

14. Dahal, R: Existence of positive solutions of a nonlinear singular semipositone dynamic equation system. Commun. Appl. Anal. 16(3), 389-402 (2012)

15. Bai, D, Xu, Y: Positive solutions for semipositone BVPs of second-order difference equations. Indian J. Pure Appl. Math. 3991, 59-68 (2008)

16. Atici, FM, Guseinov, GS: On Green's functions and positive solutions for boundary value problems on time scales, dynamic equations on time scales. J. Comput. Appl. Math. 141(1-2), 75-99 (2002)

17. Zhong, M, Zhang, $X$ : The existence of multiple positive solutions for a class of semipositone Dirichlet boundary value problems. J. Appl. Math. Comput. 38, 145-159 (2012)

18. Guo, D, Lakshmikantham, V: Nonlinear Problems in Abstract Cones. Notes and Reports in Mathematics in Science and Engineering, vol. 5. Academic Press, San Diego (1988)

19. Cabada, A, Vivero, D: Expression of the Lebesgue $\Delta$-integral on time scales as a usual Lebesgue integral: application to the calculus of $\Delta$-antiderivatives. Math. Comput. Model. 43(1-2), 194-207 (2006)

10.1186/1687-1847-2013-335

Cite this article as: Dahal: Multiple positive solutions of a semipositone singular boundary value problem on time scales. Advances in Difference Equations 2013, 2013:335

\section{Submit your manuscript to a SpringerOpen ${ }^{\ominus}$ journal and benefit from:}

- Convenient online submission

- Rigorous peer review

- Immediate publication on acceptance

Open access: articles freely available online

- High visibility within the field

- Retaining the copyright to your article 